\title{
Automated Calibration for Computerized Analysis of Prostate Lesions Using Pharmacokinetic Magnetic Resonance Images ${ }^{\star}$
}

\author{
Pieter C. Vos, Thomas Hambrock, Jelle O. Barenstz, and Henkjan J. Huisman \\ Department of Radiology, University Medical Centre Nijmegen, Netherlands \\ p.vos@rad.umcn.nl
}

\begin{abstract}
The feasibility of an automated calibration method for estimating the arterial input function when calculating pharmacokinetic parameters from Dynamic Contrast Enhanced MRI is shown. In a previous study [1], it was demonstrated that the computer aided diagnoses (CADx) system performs optimal when per patient calibration was used, but required manual annotation of reference tissue. In this study we propose a fully automated segmentation method that tackles this limitation and tested the method with our CADx system when discriminating prostate cancer from benign areas in the peripheral zone.

A method was developed to automatically segment normal peripheral zone tissue $(P Z)$. Context based segmentation using the Otsu histogram based threshold selection method and by Hessian based blob detection, was developed to automatically select $P Z$ as reference tissue for the per patient calibration.

In 38 consecutive patients carcinoma, benign and normal tissue were annotated on MR images by a radiologist and a researcher using whole mount step-section histopathology as standard of reference. A feature set comprising pharmacokinetic parameters was computed for each ROI and used to train a support vector machine (SVM) as classifier.

In total 42 malignant, 29 benign and 37 normal regions were annotated. The diagnostic accuracy obtained for differentiating malignant from benign lesions using a conventional general patient plasma profile showed an accuracy of 0.65 (0.54-0.76). Using the automated segmentation per patient calibration method the diagnostic value improved to 0.80 (0.71-0.88), whereas the manual segmentation per patient calibration showed a diagnostic performance of 0.80 (0.70-0.90).

These results show that an automated per-patient calibration is feasible, a significant better discriminating performance compared to the conventional fixed calibration was obtained and the diagnostic accuracy is similar to using manual per-patient calibration.
\end{abstract}

\section{Introduction}

Several studies have indicated that multimodal MRI increases the prostate cancer (PCa) localization accuracy of the radiologist. The accuracy is, however,

\footnotetext{
^ This work was funded by grant KUN 2004-3141 of the Dutch Cancer Society.
} 
dependent on the experience of the radiologist [234]. To help improve the diagnostic accuracy of the (unexperienced) radiologist, we are investigating the possible additional value of CADx. Previously [1, the feasibility was demonstrated of an in-house developed CADx system that calculates the malignancy likelihood of a given suspicious area in the peripheral zone of the prostate using T1-w DCE-MRI at 1.5T. Discrimination of malignant and benign regions was performed using a SVM as classifier that was trained with features extracted from quantitative pharmacokinetic (PK) maps as well as T1 estimates. The study showed that a diagnostic accuracy of 0.83 (0.75-0.92) was obtained by a standalone CADx, which is comparable to an expert radiologist performance.

Pharmacokinetic (PK) DCE-MRI could further improve PCa differentiation by reducing inter patient and inter MR scanner fluctuations compared to conventional DCE-MRI. PK tissue parameters are estimated by fitting a tracer physiologic compartment model to the observed DCE-MRI data that is driven by a plasma profile. Various techniques for estimating plasma profiles exist. Quite some PK estimators do not include per patient calibration, but use a general patient plasma profile (fixed calibration) [56]. Huisman et al. 7] demonstrated that the plasma profile varies per patient and thus, fixed calibration can cause fluctuation among patient when estimating the PK parameters. In [8], it was shown that the CADx system performs significantly better using per patient calibration instead of fixed calibration. The presented method was, however, dependent on manual annotation of healthy tissue before a malignancy likelihood could be calculated. This study addresses that limitation by presenting a more objective and automated calibration method and investigates its effect on the diagnostic accuracy of the CADx system.

The purpose of this study was to investigate the feasibility of a CADx system capable of objectively discriminating $\mathrm{PCa}$ from non-malignant disorders located in the peripheral zone of the prostate using an automated per patient calibration method.

\section{Method}

\subsection{Pharmacokinetic Modeling}

Analysis of DCE-MRI data requires knowledge of the concentration of the contrast agent in the blood plasma. Without calibration (or fixed calibration), interpatient plasma profile variability causes fluctuations in PK estimates, which are not related to the tissue condition. When using a power injector the most likely cause of differences in plasma curves are differences in body weight (total distributional volume), heart rate, vascular condition. Removing the plasma shape can be regarded as a form of patient calibration whereas fixed calibration uses a fixed plasma function over all patients.

The parametric model for analyzing contrast agent concentration time curves in DCE-MRI is the two compartment model of Tofts et al. 9. The observed concentration-time curve can be expressed as:

$$
C_{v}(t)=h\left(t ; t_{0}, V_{e}, K^{\text {trans }}, \text { Washout }\right) \otimes C_{p}(t),
$$


where $C_{v}($.$) denotes the observed tracer concentration, h($.$) the tissue impulse$ response, $C_{p}(t)$ the plasma input function and $t_{0}, V_{e}, K^{\text {trans }}$,W ashout are parameters from the model. The reference tissue method estimates the plasma input function by:

$$
\hat{C}_{p}(t)=C_{r e f, v}(t) / h_{r e f, v}(t),
$$

where $C_{r e f, v}($.$) represents the observed plasma profile for tissue v$ and $h_{r e f, v}($. a reference plasma profile for tissue $v$ based on literature. The reference tissue method is considered to be a robust technique [10.

\subsection{Automated Per Patient Calibration}

In a previous study 8 , it was demonstrated that using $P Z$ as reference tissue gave good results for estimating PK parameters. In this study a method was developed to auto segment $P Z$. The method is divided into two stages. First, the location of the prostate is detected using a blob detection method. In the second stage, this location is further refined to segment a $P Z$ region.

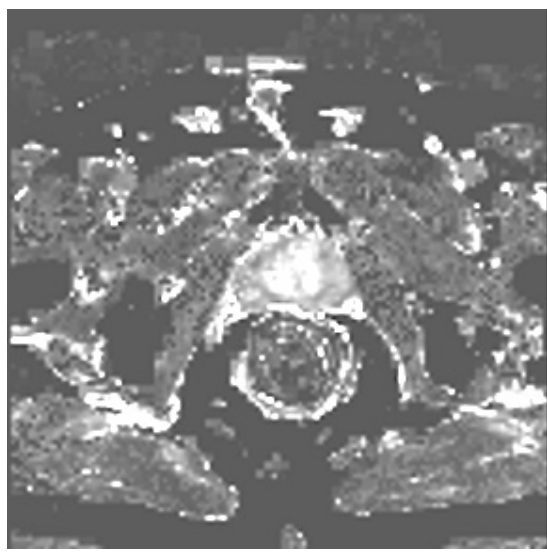

(a) DCE of the prostate area.

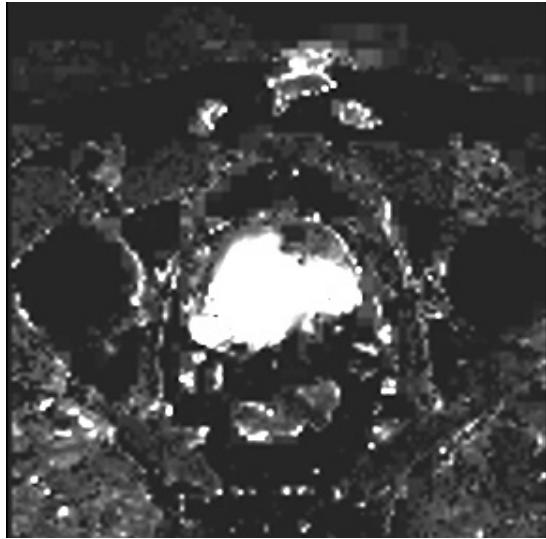

(b) DCE of the bladder area.

Fig. 1. Rational for modeling an early and large enhancing blob in the pelvic area

Automated Localization of the Prostate. The prostate can be modelled as a large enhancing area (or blob) in the pelvis. Figure 1(a) demonstrates this model where the prostate can easily be detected by a human observer. Large and strong enhancements can be observed in the transition zone of the prostate making it suitable for detection. First experimental results showed however, that this assumption is not only true for the prostate. Because the acquisition time for the DCE-MRI can be rather long (3 min), contrast agent also arrives in the bladder, resulting in a comparably large enhancing blob, as demonstrated in figure $1(\mathrm{~b})$. The prostate model is therefore extended by including the arrival time of the contrast agent $\left(t_{0}\right.$ of $\left.C_{v}(t)\right)$. Otsu's automatic threshold selection 
method from gray-level histograms ([11]) is used to segment early enhancing structures in the relative enhancement image $V(x)$ :

$$
V_{O}(\mathbf{x})=\left\{\begin{array}{l}
V(\mathbf{x}), \quad t_{0}(x)<t h_{O t s u} \\
0
\end{array}\right.
$$

A common approach to detect blobs is to consider the Taylor expansion of $V_{O}$ at multiscale for a given neighborhood of pixel $x$ [12,

$$
V_{O}(\mathbf{x}+\delta \mathbf{x}, \sigma) \approx V_{O}(\mathbf{x}, \sigma)+\delta \mathbf{x}^{T} \nabla_{\sigma}+\delta \mathbf{x}^{T} H_{\sigma} \delta \mathbf{x}
$$

where $\nabla_{\sigma}$ and $H_{\sigma}$ are the gradient vector and Hessian vector of an image at scale $\sigma$. Here, $V_{O}$ is convolved using derivatives of Gaussians:

$$
\frac{\delta}{\delta \mathbf{x}} V_{O}(\mathbf{x}, \sigma)=\sigma V_{O}(\mathbf{x}) \frac{\delta}{\delta \mathbf{x}} G(\mathbf{x}, \sigma)
$$

Next, from $H_{\sigma}$ eigenvalues $\lambda_{\sigma, k}$ are computed, corresponding the the $\mathrm{k}$-th normalized vector $\hat{u}_{\sigma, k}$ and analyzed to determine the likelihood of a pixel $\mathbf{x}$ belonging to a blob. This analysis is based on the following likelihood function (for bright blob, dark background):

$$
P(\mathbf{x}, \sigma)=\left|\lambda_{1}(x)\right|\left|\lambda_{2}(x)\right|\left|\lambda_{3}(x)\right|
$$

that is, all three eigen values should be large to represent a blob. A multiscale approach is adopted after which the maximum response is selected:

$$
P(\mathbf{x})=\max _{\sigma_{\min } \leq \sigma \leq \sigma_{\max }} P(\mathbf{x}, \sigma)
$$

The center location of the prostate $x_{p c}$ containing the highest probability is then selected by $x_{p c}=\arg \max _{x} P(\mathbf{x})$. In figure 2(a) a probability map is shown that is used for the prostate detection.

Automated Segmentation of Normal Peripheral Zone Tissue. In the second stage of the method, a context based segmentation is performed to extract normal peripheral zone tissue. The method is based on the model that the $P Z$ is mainly dorsal located of $x_{p c}$. Thus, we define a box-mask below $x_{p c}$ with height, width and depth set to $\sigma$ to mask $V_{O}$. Here, $\sigma$ corresponds with the size of the prostate and is the scale at which $P\left(x_{p c}\right)$ was found by the blob detector:

$$
S\left(x_{p c}\right)=\arg \max _{\sigma}\left(P\left(x_{p c}, \sigma\right)\right.
$$

Figure 2(b) demonstrates this model. Simple thresholding of extrema and removal of sharp edges using a gradient magnitude filter can now be applied to the box-mask which results in the segmentation of normal peripheral zone tissue, as demonstrated in figures $2(\mathrm{c})$ and $2(\mathrm{~d})$. 


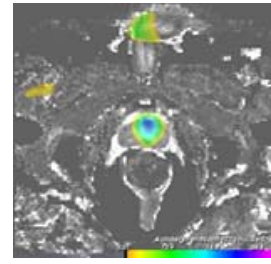

(a) $S\left(x_{p c}\right)$

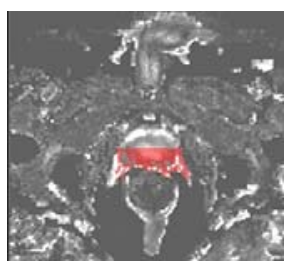

(b) Model of $P Z$

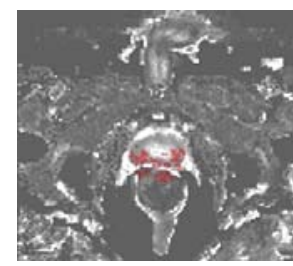

(c) Strong gradient(d) Extrema threshremoval

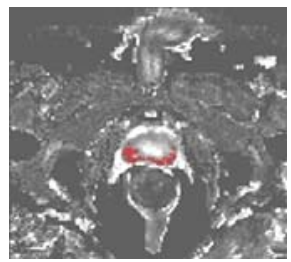

olding

Fig. 2. Example case of context based segmentation of normal peripheral zone tissue

\subsection{CADx Performance Evaluation}

The features $K^{\text {trans }}, V_{e}$ and Washout were computed and used to train a SVM as classifier [9]. The features were combined into a single malignancy likelihood estimate using the SVM. The output of the classifier was used as a measure of likelihood of malignancy. The discriminating performance of the CADx system was estimated by means of the area under the receiver operator characteristics (ROC) curve (AUC). The prospective performance of the lesion analysis with per patient and fixed calibration were estimated by means of leave-one-patientout (LOPO) cross validation. LOPO avoids training and testing on the same data and to emphasize the prospective value, one whole patient case was drawn from the set. The LOPO involves training on all but one case, estimating the likelihood of that left-out case, and repeating the procedure until each case has been tested individually. The bootstrap technique was used to compute $95 \%$ confidence intervals for the AUC and significance level for the paired difference [13.

\subsection{Experiment}

The study set consisted of 38 consecutive patients that were selected between January 2007 and October 2008. These patients had biopsy-proven PCa and underwent dynamic contrast-enhanced MR imaging at 3.0T, complementary to the routine staging MR imaging examination of the prostate. Patients were included in the study only if they were candidates for radical retropubic prostatectomy within 6 weeks after MR imaging. The study was approved by the institutional review board, and informed consent was obtained from all patients prior to MR imaging. Exclusion criteria were: previous hormonal therapy, lymph nodes positive for metastases at frozen section analysis, contraindications to MR imaging (e.g., cardiac pacemakers, intracranial clips), contraindications to endorectal coil insertion (e.g., anorectal surgery, inflammatory bowel disease).

Images were acquired with a 3.0T whole body MR scanner (TrioTim, Siemens Medical Solutions, Erlangen, Germany). A pelvic phased-array as well as a balloon-mounted disposable endorectal surface coil (MedRad@), Pittsburgh, PA, USA) inserted and inflated with approximately $80 \mathrm{~cm}^{3}$ of Perfluorocarbon (FOMBLIN LC08), were used for receiving. The machine body coil was used for 
RF transmitting. An amount of $1 \mathrm{mg}$ of glucagon (Glucagonß), Novo Nordisk, Bagsvaerd, Denmark)) was administered directly before the MRI scan, to all patients to reduce peristaltic bowel movement during the examination.

High-spatial-resolution T2-weighted fast spin-echo imaging in the axial, sagittal and coronal planes, covering the prostate and seminal vesicles, was performed. 3D T1-weighted spoiled gradient echo images were acquired before and during an intravenous bolus injection of paramagnetic gadolinium chelate $(0.1 \mathrm{mmol} / \mathrm{kg}$, gadopentetate, Magnevist_R; Schering, Berlin, Germany) using a power injector (Spectris, MedradR), Pittsburgh, PA, US) with an injection rate of $2.5 \mathrm{ml} / \mathrm{second}$ followed by a $15 \mathrm{ml}$ saline flush for $300 \mathrm{sec}$ every 3 seconds. Fitting the DCE-MRI is decribed elsewhere 14.

Whole-mount step-section histology tumor maps were used as ground truth for annotating $\mathrm{PCa}$ (with a relevant diameter of at least $5 \mathrm{~mm}$ ), non-malignant suspicious enhancing (NS) and normal (N) regions on T2-w images for all patients in consensus by two readers.

\section{Results}

One patient case was excluded because the DCE examination had failed. In total 42 malignant regions were annoted in the peripheral zone. The number of NS regions annotated in the peripheral zone was 29. The number of normal peripheral zone regions was 38 .

The effect of the per patient calibration on the diagnostic performance was first evaluated by pairwise scatterplots of $\mathrm{PK}$ parameters of the lesions. It is noticeable in figure 3(a) that without calibration the clusters overlap more than the clusters in figure 3(c) where manual calibration is included. Figure 3(b) shows similar results for automatic patient calibration. Furthermore, the $N$ and $N S$

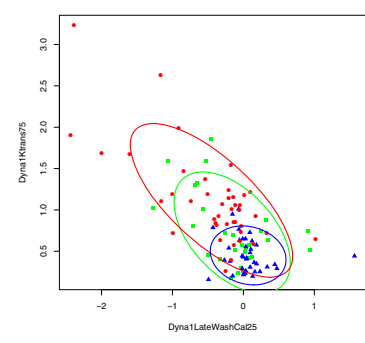

(a) Fixed

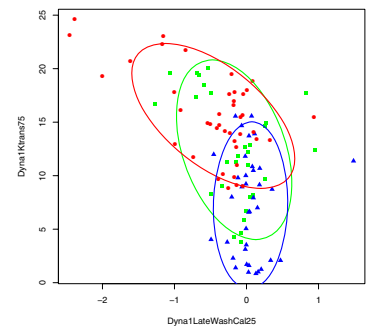

(b) Auto

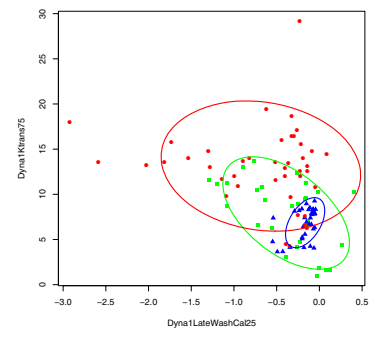

(c) Manual

Fig. 3. Pairwise scatterplots of 2 kinetic parameters, Washout versus $K^{\text {trans }}$, for the whole database with squares representing NS regions, spheres as malignant regions and triangles as $\mathrm{N}$ regions for the different calibration methods used. The ellipsoids summarize the three clusters by fitting a bivariate normal distribution and displaying the outline at 2 times standard deviation radius. It is noticeable that the clusters overlap one another when fixed calibration is used, whereas manual and automated per patient calibration demonstrate a noticeable clustering of features. 


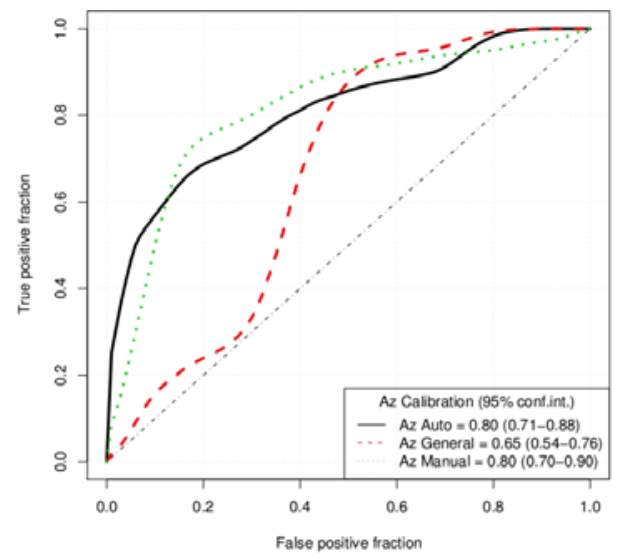

Fig. 4. ROC curves showing the discriminating performance of the CADx system using the different calibration methods fixed, automated per patient and manual per patient calibration

clusters have a smaller covariance when patient calibration is used. An effect on the diagnostic performance can therefore be expected. The different distributions demonstrate the strong effect of the chosen calibration method.

The performance of discriminating malignant lesions from NS areas with fixed, manual and automatic calibration is demonstrated in the ROC curves shown in figure 3. Here, the focus is on the characterization of NS and malignant regions, because it is more challenging and clinically relevant. The diagnostic accuracy was $0.65(95 \%$ confidence intervals $=0.54-0.76))$ when fixed calibration was used. The diagnostic accuracy improved significantly for both manual per patient calibration, $\mathrm{Az}=0.80$ (0.70-0.90), as for automated per patient calibration, $\mathrm{Az}=0.80$ (0.71-0.88). The marginal difference between the automated and manual calibration means that they perform similar, which was the intended goal.

\section{Conclusion}

In this study, we have demonstrated the feasibility of an automated calibration method for estimating the arterial input function when calculating pharmacokinetic parameters from DCE-MRI. The results show a significant better discriminating performance $(\mathrm{Az}=0.80(0.71-0.88))$ compared to the conventional fixed calibration. The performance is similar to using the manual per patient calibration.

\section{References}

1. Vos, P., Hambrock, T., Hulsbergen-van de Kaa, C., Fütterer, J., Barentsz, J., Huisman, H.: Computerized analysis of prostate lesions in the peripheral zone using dynamic contrast enhanced MRI. Med. Phys. 35(3), 888-899 (2008)

2. Hricak, H., Choyke, P., Eberhardt, S., Leibel, S., Scardino, P.: Imaging prostate cancer: a multidisciplinary perspective. Radiology 243(1), 28-53 (2007) 
3. Fütterer, J., Heijmink, S., Scheenen, T., Veltman, J., Huisman, H., Vos, P., de Kaa, C., Witjes, J., Krabbe, P., Heerschap, A., Barentsz, J.: Prostate Cancer Localization with Dynamic Contrast-enhanced MR Imaging and Proton MR Spectroscopic Imaging. Radiology 241(2), 449-458 (2006)

4. Chan, I., Wells, W., Mulkern, R., Haker, S., Zhang, J., Zou, K., Maier, S., Tempany, C.: Detection of prostate cancer by integration of line-scan diffusion, T2-mapping and T2-weighted magnetic resonance imaging; a multichannel statistical classifier. Med. Phys. 30(9), 2390-2398 (2003)

5. Weinmann, H.J., Laniado, M., Mutzel, W.: Pharmacokinetics of GdDTPA/ dimeglumine after intravenous injection into healthy volunteers. Physiol. Chem. Phys. Med. NMR 16(2), 167-172 (1984)

6. Brix, G., Semmler, W., Port, R., Schad, L.R., Layer, G., Lorenz, W.J.: Pharmacokinetic parameters in CNS Gd-DTPA enhanced MR imaging. J. Comput. Assist. Tomogr. 15(4), 621-628 (1991)

7. Huisman, H., Veltman, J., Boetes, C., Karssemeijer, N., Blickman, H., Barentsz, J.: Computer-aided diagnosis of breast MRI using pharmacokinetic modeling. RSNA, SSC17-09 (2006)

8. Vos, P.C., Hambrock, T., Fütterer, J.J., van de Kaa, C.A.H., Barentsz, J., Huisman, H.H.: Effect of calibration on computerized analysis of prostate lesions using quantitative dynamic contrast-enhanced magnetic resonance imaging. In: SPIE, vol. 6514, 65140U (2007)

9. Tofts, P., Brix, G., Buckley, D., Evelhoch, J., Henderson, E., Knopp, M., Larsson, H., Lee, T., Mayr, N., Parker, G., Port, R., Taylor, J., Weisskoff, R.: Estimating kinetic parameters from dynamic contrast-enhanced T(1)-weighted MRI of a diffusable tracer: standardized quantities and symbols. J. Magn. Reson. Imaging 10(3), 223-232 (1999)

10. Kovar, D., Lewis, M., Karczmar, G.: A new method for imaging perfusion and contrast extraction fraction: input functions derived from reference tissues. J. Magn. Reson. Imaging 8(5), 1126-1134 (1998)

11. Otsu, N.: A threshold selection method from gray-level histograms. EEE Trans. Sys., Man., Cyber. 9(1), 62-66 (1979)

12. Frangi, A.F., Niessen, W.J., Vincken, K.L., Viergever, M.A.: Multiscale vessel enhancement filtering. In: Wells, W.M., Colchester, A.C.F., Delp, S.L. (eds.) MICCAI 1998. LNCS, vol. 1496, p. 130. Springer, Heidelberg (1998)

13. Rutter, C.: Bootstrap estimation of diagnostic accuracy with patient-clustered data. Acad. Radiol. 7(6), 413-419 (2000)

14. Hittmair, K., Gomiscek, G., Langenberger, K., Recht, M., Imhof, H., Kramer, J.: Method for the quantitative assessment of contrast agent uptake in dynamic contrast-enhanced MRI. Magn. Reson. Med. 31(5), 567-571 (1994) 